\title{
EDITORIAL
}

\section{Electromagnetic compatibility}

Electromagnetic Compatibility, internationally known for its acronym from English as EMC, is defined as the ability of a device, equipment or system to function satisfactorily in its electromagnetic environment without causing intolerable electromagnetic disturbances to anything in that environment. It is clear that any device, equipment or system that requires electrical energy power for operation, is immersed in an environment of electromagnetic energy radiated or guided, where radiated electromagnetic energy is the one propagating through free space and guided electromagnetic energy is the one that propagates in a physical medium such as a solid conductive material, a hollow conductor material or a dielectric material. In order to comply with the requirements of the EMC, the limits of radiated and conducted electromagnetic energy need to be known, in order to prevent the generation of disturbance or being susceptible to such energies. The determination of these limits was set after analyzing several investigations, which were carried out in universities, research institutes, industrial laboratories, etc. Results were discussed by international committees and from the results, the limits were set for electromagnetic emissions. Currently, data of those limits are specified in international or local standards, the most common are: IEC, IEEE, ANSI, STD, CISPR FCC, CENELEC, among others.

In this regard, the EMC is focused on three key issues: (1) To ensure that the electromagnetic signals in their path are not affected by electromagnetic interference, (2) To prevent the generation of electromagnetic interference and (3) To minimize susceptibility to electromagnetic interference. The first issue focuses on the problems of the propagation and transmission medium. The second issue is concerned with avoiding the generation of electromagnetic energy that degrades the performance of systems, equipment and electrical and electronic devices. The mentioned phenomena may be avoided or minimized by filtering, shielding or by appropriate distribution of components in systems or equipment. The third issue deals with the problem known as electromagnetic interferences (EMI), that are defined as the degradation of the performance of a device, equipment or system caused by an electromagnetic disturbance. The fact that EMI degrades the performance of systems, devices or equipment operating with electric power, indicates that they are susceptible to such disturbances because they have a high level of sensitivity. To minimize this sensitivity, it is necessary to design robust electrical/electronic systems, for them to function without degradation. Radio communication systems are the most critical subject to the effects of radiated electromagnetic emissions, due to their high sensitivity, since they operate in natural or man-made electromagnetic environments. Some electronic equipment such as transmitters, are designed to transmit RF power within a frequency range, which must be received by an antenna of a radio receiver, in this case the radiated electromagnetic energy is intentional. Intentional signals of radiated electromagnetic energy are useful for certain systems and for others they become undesirable, because they lead to disturbances.

To understand all aspects of Electromagnetic Compatibility, some universities in the world have proposed EMC courses for electrical, electronics and telecommunications engineers, as well as biomedical engineering and in computer systems. EMC education has many challenges, due to the fact that it involves phenomena which are analyzed, from classical electromagnetic theory, for the design of analog and digital electronic circuits with low and high integration, as well as development of computer models to simulate all effects that can cause electromagnetic interference. To understand all the aspects that are presented with the electromagnetic interference (EMI), several universities have begun to prepare specialists capable of working on solving problems regarding: 
(1) PCB, Printed Circuit Board Design: In order to minimize the EMI problems in the PCB design, it is necessary to consider all EMC aspects, being the most important the optimal distribution of components with appropriate connecting paths and ground systems. These design techniques are important in high frequency, because physical length of the devices is equal or similar to the wavelength of the signal that is processed, where crosstalk problems are more common;

(2) Filter Design: The function of a filter is to eliminate or minimize the EMI (electromagnetic energy) in the form of current or voltage, when it is conducted through interconnections in electrical and electronic systems. In the UHF frequency band and microwaves, the filters with EBG structures (Electromagnetic Band Gap) and metamaterials have allowed to optimize the signal integrity, because these filters are built in the same substrate of the PCB;

(3) Electromagnetic shielding and grounding systems: The radiated electromagnetic energy may disturb any device, equipment or system, so it is necessary to provide shielding for this kind of energy. The shielding and ground systems can be made from the connections (wiring) up to the shielding of the room, such as the Faraday cages. An adequate grounding system reduces the sensitivity to electromagnetic interferences. The type of shielding and grounding system depends on the application;

(4) Measurements of aspects related to electromagnetic compatibility. If an evaluation, from a viewpoint of electromagnetic compatibility is required, a procedure must be followed, that normally specifies the standards. However, due to the large number of devices, equipment and systems operating with electric power and that every day they become more integrated into the daily life, testing and preset limits for the EMC are exceeded, so we need to have new proposals new for the limits, as well as measurement systems for conformity evaluation of electromagnetic compatibility;

(5) Electromagnetic energy sensors: In the topics of EMC, these kinds of sensors are essential to identify the electromagnetic emissions, which may cause disturbances in all radio electric spectrums. With these sensors it is possible to evaluate the radiated and conducted electromagnetic emissions, which represent the first element of a system for the conformity evaluation of the EMC. The challenge is the development of new sensors for application in these issues, with the goal to achieve the highest bandwidth, their versatility in physical dimensions and calibration facility to provide certainty in the measurement processes;

(6) Evaluation models for electromagnetic compatibility: In general, most of the problems in EMC topics are complex and of random nature. To evaluate such problems, statistical techniques and probabilistic models must be applied. For this evaluation, the development of numerical methods to simulate systems and analyze the phenomena that occur in the processes of electromagnetic disturbances is also required.

For several years in Mexico, institutions providing postgraduate studies in electric engineering offer courses with the topic of EMC, but not exclusively of this subject. The National Polytechnic Institute, at Superior School of Mechanical and Electric Engineering, Campus Zacatenco (IPN-ESIME-ZAC), in the Electronic Engineering Postgraduate Programme teaches theoretical-experimental EMC courses, exclusively about this subject. The non-ideal component analysis; the integrity signal; radiated and conducted electromagnetic energy, which cause EMIs; the electromagnetic shielding, the grounding plane systems and others, are fundamental topics of the EMC courses. The aforementioned EMC group of the Postgraduate Programme has the responsibility of the courses as well as the development of their experimental component. The EMC group has an Electromagnetic Compatibility Laboratory with a precomformance anechoic chamber, a GTEM cell, antennae, network vector analyzers, EMI test receiver, power amplifier, oscilloscopes and other instruments to carry out experimental research about EMC. The most significant projects in recent years are: (1) Electromagnetic Compatibility Analysis of power grids in Mexico, to install broadband communications, known as PLC (Power Line Communication); (2) Evaluation of RFID technology applied to the identification of all motor vehicles transiting in the Mexican Republic; (3) Development of electromagnetic field sensors (antenna) ultrabroadband, using metamaterials with multilayer substrates and conductive plastics for application to 
portable instruments for measuring electromagnetic interferences. The EMC laboratory made it possible to train human resources in an important area of development for any country, their graduates being absorbed by the domestic industry with international competitiveness. Now EMC plays a key role in the contemporary world in all areas of daily life, so it is fundamental to give it equal attention in engineering curriculum. Also, EMC has important influences on living organisms, as they are affected by electromagnetic fields, thus this is also a topic that should be discussed in the field of medicin.

Roberto Linares y Miranda

José Luis López Bonilla

Compatibility Electromagnetic Laboratory

Superior School of Mechanical and Electrical Engineering,

SEPI-Zacatenco

National Polytechnic Institute,

México DF, México

rlinaresy@ipn.mx; jlopezb@ipn.mx 